\title{
Embarazo abdominal calcificado con 44 años de evolución
}

\author{
Isabella Carvalho Oliveira ${ }^{a}$, Pablo Henrique Coelho Bringela , Denise Borba Galdino ${ }^{\text {, }}$ \\ Anselmo Fernandes Rezende de Oliveira ${ }^{a}$, Viviane Tiemi Kenmoti ${ }^{1}$, Luciano Augusto de \\ Pádua Fleury Neto $^{2}$, Gesneria Saraiva Kratka. ${ }^{3}$ \\ ${ }^{1}$ Servicio de Cirugía, Hospital Regional de Porto Nacional; ${ }^{2}$ Servicio de Imagenología, Hospital General de Palmas; \\ 3 Servicio de Obstetricia y Ginecología, Hospital Regional de Porto Nacional. Brasil.
}

a Alumna/o, Escuela de Medicina, Universidad Federal de Tocantins. Brasil.

\section{RESUMEN}

Lithopedion (litho = piedra, pedion $=$ niño), es el término utilizado para describir un feto intrabdominal calcificado. Es un evento raro, con aproximadamente 300 casos descritos en la literatura internacional. Este artículo trata del caso de una mujer de 84 años con dolor abdominal, donde se evidenció en el examen físico, una masa abdominal de consistencia pétrea, con dimensiones de $23 \mathrm{~cm}$ de altura y $32 \mathrm{~cm}$ de longitud. El examen radiológico del abdomen reveló la presencia de imagen de densidad ósea, sugiriendo la existencia de un feto abdominal calcificado. La tomografía computarizada confirmó tratarse de un litoquelifopedion de 29-30 semanas, con aproximadamente 44 años de evolución.

\section{PALABRAS CLAVE: Lithopedion, feto, tumor abdominal, litoquelifopedion}

\section{SUMMARY}

Lithopedion (litho $=$ stone, pedion $=$ child) is the term used to describe an intra-abdominal calcified fetus. In the international literature, about 300 cases were described. In this case, an 84-year old woman was admitted with abdominal pain and the abdominal physical examination showed a mass with hard consistency with $23 \mathrm{~cm}$ height and $32 \mathrm{~cm}$ width. The abdominal x-ray examination showed the presence of bone density image suggesting an intra-abdominal calcified fetus. The computer tomography confirmed that it was a litoquelifopedion of 29-30 weeks of pregnancy and with about 44 years of evolution.

\section{KEY WORDS: Lithopedion, fetus, abdominal tumor, litoquelifopedion}

\section{INTRODUCCIÓN}

La gestación ectópica es una complicación que puede ocurrir en el primer trimestre del embarazo y es una causa frecuente de dolor abdominal agudo (1). En el $95 \%$ de los casos de gestación ectópica la implantación ocurre en las tubas y el $5 \%$ restantes en los ovarios, en el canal cervical, en la cicatriz de cesárea o en la cavidad peritoneal (2).
La implantación intrabdominal del feto generalmente resulta en la muerte del concepto por insuficiencia placentaria $(3,4)$. Ese contenido puede ser nuevamente absorbido o dar inicio a un proceso de deshidratación, momificación y calcificación, formando un lithopedion (litho $=$ piedra, pedion = niño). Esa condición ocurre en $0,0054 \%$ de todas las gestaciones y en $1,5 \%$ al $1,8 \%$ de las gestaciones abdominales $(5,6)$. 
En la literatura internacional, durante los últimos 400 años, fueron descriptos más de 300 casos de lithopedion $(5,7,8)$. El relato médico más antiguo tiene fecha del siglo $X$ y comunicado por el cirujano árabe, Albucasis, en una enciclopedia médica árabe (9). En el Brasil, desde 1983 hasta el momento, se publicaron (indexados a la Biblioteca Virtual en Salud) cinco casos de litopedion, siendo el más antiguo de 1983 (10).

En virtud de lo raro y de la importancia del embarazo ectópico y de su evolución hacia la calcificación, describimos un caso de lithopedion retenido por 44 años en una paciente de 84 años de edad.

\section{Caso clínico}

Mujer de 84 años, viuda, que nació y vive en Natividad, Tocantins, Brasil. La paciente se registró en el Hospital Regional de Porto Nacional, Tocantins, por recomendación del Hospital Regional de Natividad, Tocantins, con histórico de epigastralgia de 15 días de evolución, de carácter persistente e intenso, que calma con el uso de tés caseros (naranja y hierba cidrera) y sin elementos de agravamiento. Asociado a ello, reportó episodios eméticos y distensión abdominal. Niega presencia de fiebre, alteraciones de hábitos intestinal y urinario. Relata que estos episodios eran frecuentes y recurrentes desde hace más de 40 años. La persistencia de estos síntomas hizo que buscara atención médica, y se constató masa abdominal de consistencia pétrea. Se realizó radiografía de abdomen y fue enviada a Porto Nacional, Tocantins, para aclaración del diagnóstico.

La paciente reportó que hace 44 años pasó por un periodo de amenorrea por más de tres ciclos consecutivos, siendo que antes poseía ciclos menstruales regulares y no hacía uso de métodos contraceptivos. Durante ese periodo notó el aumento del volumen abdominal y el surgimiento de movimientos fetales similares a las gestaciones anteriores, pero no procuró atención médica. Además, presentó episodios de dolor abdominal intenso. El más importante ocurrió con dolor en puntada en fosa iliaca izquierda que irradiaba difusamente por el abdomen, asociado a náuseas, astenia, hiporexia y síncope. Esa condición llevó a la paciente a quedarse acostada en su casa durante más de 15 días. Después del uso de medicinas caseras durante 30 días, volvió el ciclo menstrual regular y hubo mejoría en los síntomas reportados. Sin embargo, después de algún tiempo, la paciente notó la aparición de una masa abdominal de consistencia pétrea y la ausencia de los movimientos fetales que había notado antes.
La paciente relató que tuvo dos embarazos anteriores, con hijos nacidos vivos de partos normales. En los antecedentes personales patológicos la paciente negó comorbilidades y cirugías previas. En el momento del examen físico abdominal, la paciente presentó a la inspección un abultamiento que se extendía, longitudinalmente, del hipogastrio hasta aproximadamente $10 \mathrm{~cm}$ arriba de la cicatriz umbilical y, transversalmente, de la fosa ilíaca derecha (FID) hasta la fosa ilíaca izquierda (FIE), con compromiso de la porción superior del flanco izquierdo. Durante la auscultación abdominal se observó la ausencia de ruidos hidro-aéreos en toda la región abdominal izquierda. En la percusión se observó que la región entre FID y FIE era sólida. La palpación evidenció masa de consistencia pétrea, con dimensiones de $23 \mathrm{~cm}$ de altura y $32 \mathrm{~cm}$ de longitud. Al examen ginecológico, presenta útero en retroversión, central, con paredes lisas, consistencia levemente aumentada, de tamaño y volumen normales para la edad. Colon uterino de proporciones y consistencia normales para la edad y orificio externo cerrado.

Se realizaron radiografías del abdomen (Figura 1), ultrasonografía y tomografía computarizada (Figura 2, 3, 4 y 5), que evidenciaron la presencia de feto intraabdominal calcificado.

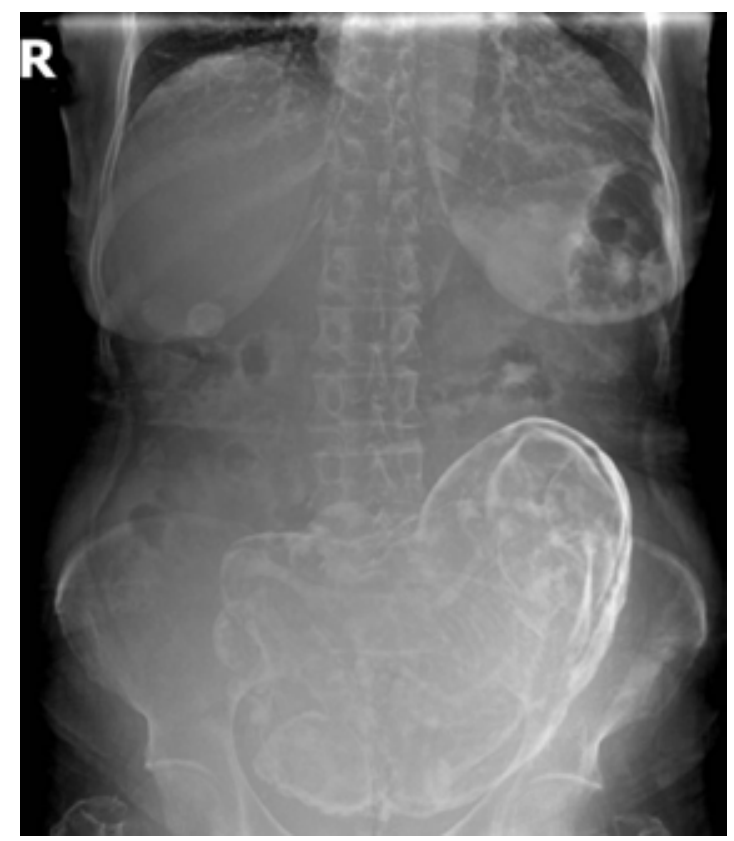

Figura 1. Radiografía que muestra feto y las membranas del saco amniótico calcificadas en posición longitudinal y presentación pélvica. 


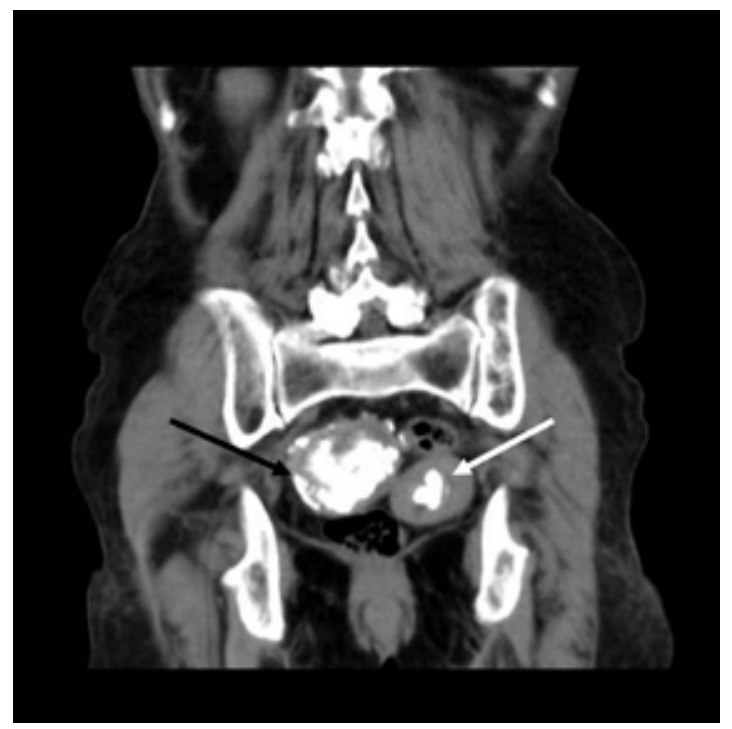

Figura 2. Tomografía computarizada, corte coronal, ventana de partes flexibles, evidenciando la presencia de imágenes calcificadas groseras, siendo una intrauterina (flecha blanca) y otra parauterina a la derecha (flecha negra).

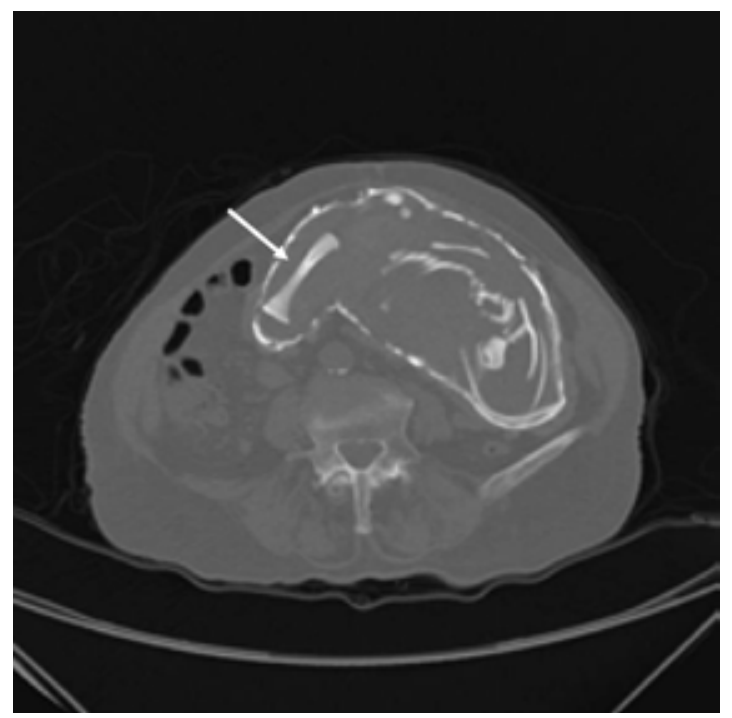

Figura 3. Tomografía computadorizada, corte axial, ventana de hueso. Presencia de saco gestacional intraabdominal de paredes calcificadas cuyo feto se encuentra en situación longitudinal y presentación pélvica, edad gestacional estimada de 29-30 semanas según medidas óseas. El fémur se visualiza longitudinalmente (flecha blanca).

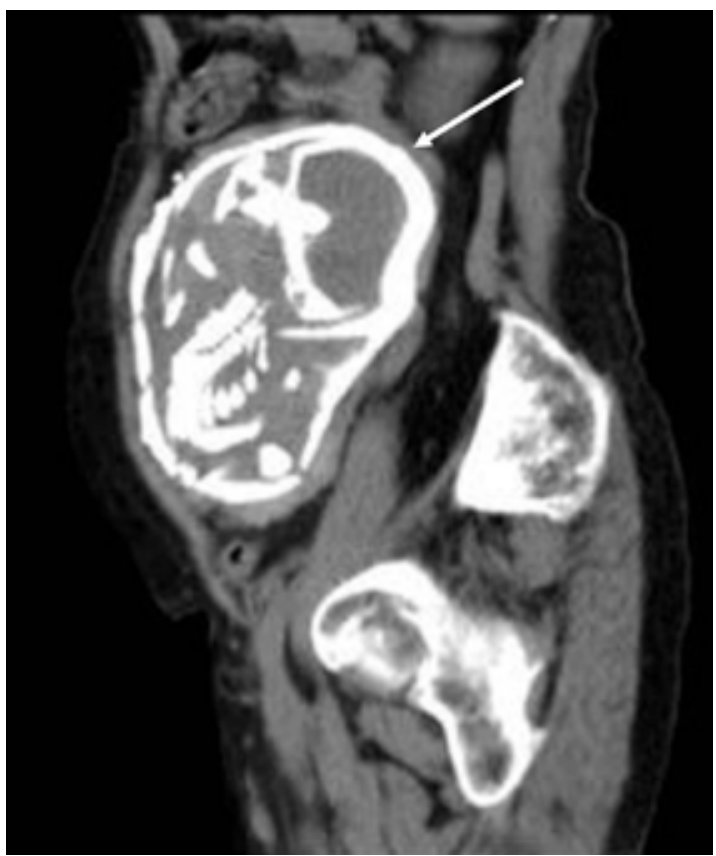

Figura 4. Tomografía computarizada, corte sagital, ventana de partes flexibles. Presencia de saco gestacional intraabdominal de paredes calcificadas (flecha blanca) cuyo feto se encuentra en situación longitudinal y presentación pélvica con edad gestacional estimada en 29-30 semanas.

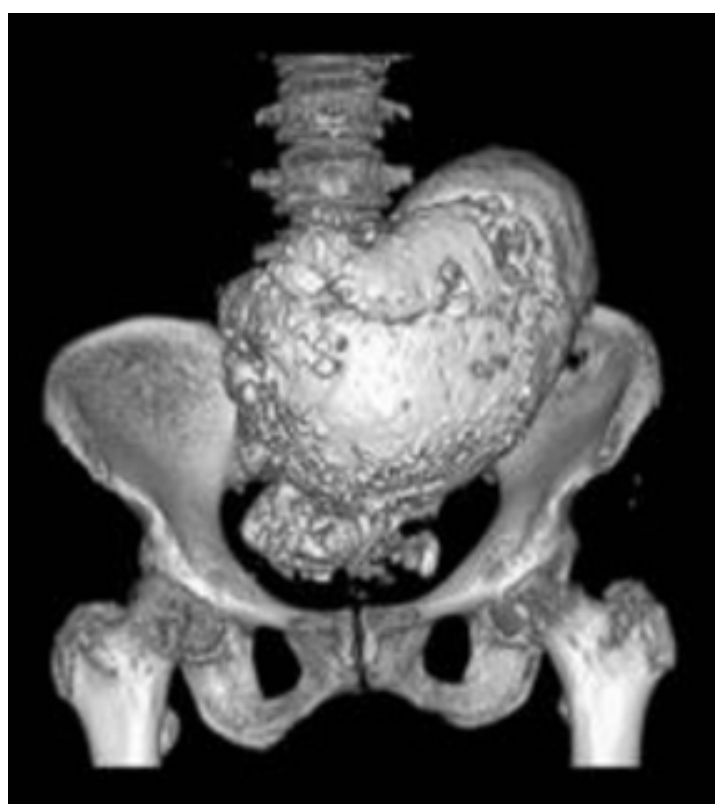

Figura 5. Tomografía computarizada con reconstrucción en 3D. Visualización del feto en situación longitudinal y presentación pélvica en la cavidad abdominal. 


\section{DISCUSIÓN}

El lithopedion resulta de un embarazo intrabdominal avanzado, donde el feto queda retenido en la cavidad abdominal materna y sufre deshidratación y calcificación (11). De acuerdo con las estructuras calcificadas, los litopediones pueden clasificarse en tres tipos: a. lithoquelifo (26\%), cuando hay sólo calcificación de las membranas, b. lithoquelifopedion $(31 \%)$ cuando el feto y las membranas están calcificadas, y lithopedion verdadero (34\%) cuando sólo el feto se encuentra calcificado (11).

Para que ocurra la gestación intrabdominal calcificada es necesario que los siguientes eventos estén presentes: embarazo extrauterino, donde la muerte fetal ocurre después de las 13 semanas de gestación, que ese feto sea estéril, que haya condiciones propicias para la deposición de calcio y que el embarazo no sea diagnosticado y tratado (11). Algún tiempo después de la muerte fetal, se inicia el proceso de petrificación (11).

El tiempo de retención fetal varía, de acuerdo con la literatura, entre 4 y 60 años, con promedio de 25 años $(11,12)$. En $43 \%$ de los casos de lithoquelifopediones, el feto tiene características de 3740 semanas de edad gestacional, mientras que en $27 \%$ de los casos el feto presenta edad gestacional entre 28 y 32 semanas, y en $20 \%$ entre 12 y 24 (11).

Relatos publicados sugieren que el embarazo abdominal avanzado está invariablemente asociado a bajas condiciones socioeconómicas (falta de recursos e instrucción) y acceso precario a cuidados prenatales $(13,14)$. En la época del surgimiento de los primeros síntomas gestacionales, la paciente no contaba con asistencia médica, lo que la llevó a buscar "curanderos" para resolución de su problema.

Lithopediones son, generalmente, asintomáticos, sin embargo, pueden llevar al surgimiento de dolor pélvico, malestar abdominal, síntomas comprensivos (urinarios o gastrointestinales), y una serie de complicaciones que abarcan desde infecciones, necrosis, abscesos pélvicos, fístulas, perforación rectal y vesical, eliminación de partes fetales a través de la pared abdominal, recto o vagina, como también obstrucción y vólvulo intestinal $(2,8,11,15)$.

En ese caso, la paciente presentó como síntoma un dolor epigástrico crónico recurrente por 44 años. En los 15 días anteriores a la internación, ese dolor evolucionó con empeoramiento asociado a distención abdominal, náuseas y vómitos, pero fue constatado posteriormente que se trataba de un cuadro de colelitiasis. A pesar del tiempo prolongado de retención del feto, la paciente no presentó ninguna complicación citada en la literatura.

El diagnóstico de lithopedion ocurre, casi siem- pre, accidentalmente por palpación de la masa abdominal durante el examen físico, por radiografía abdominal o cirugía realizados por otros motivos o durante la necropsia (11). Los exámenes que pueden ser solicitados para confirmar el diagnóstico son: 1) Radiografía de abdomen: es suficiente para la confirmación de esa condición (Figura 1) (11) En el caso reportado, la radiografía del abdomen reveló la presencia de imagen de densidad ósea con señales de calota craneana, fémures y huesos del miembro superior, arcos costales débiles, delineamiento de la columna cervical, torácica, lumbar y sacral. 2) Tomografía computarizada (TC) y Resonancia Magnética (RM), las que definen claramente la patología y ayudan en la programación de la estrategia terapéutica, en la pesquisa de adherencias y de compromiso de otros órganos. Sin embargo, esos exámenes no son absolutamente necesarios $(2,8,11,15)$. Cuando se hizo la TC se evidenció la presencia de saco gestacional intra abdominal de paredes calcificadas cuyo feto se encontraba en situación longitudinal y presentación pélvica con edad gestacional estimada en 29-30 semanas a través de medidas óseas (Figuras 3, 4 y 5). De acuerdo con este examen, el caso fue clasificado en lithoquelifopedion, debido a la presencia de feto y de saco gestacional intraabdominal calcificados. No fueron demostradas complicaciones como adherencia intestinal o compresión de órganos abdominales.

Fue posible evidenciar también la presencia de imágenes calcificadas groseras, una intrauterina y otra parauterina a la derecha (Figura 2). El equipo no logró concluir con precisión si esa última se trataba de otro feto o de partes de lo que se describe arriba. Para dicha precisión sería necesaria una laparotomía exploratoria, pero, en virtud de las condiciones clínicas de la paciente, agravadas en virtud de tratarse de una anciana, se refutó esa opción.

Hay una divergencia en la literatura sobre el tratamiento en casos de lithopedion asintomático. Algunos autores consideran que la intervención por intermedio de cirugía en esos casos es necesaria, pero otros no observaron efectos indeseables en pacientes que no se sometieron a ese procedimiento (15). La morbilidad aumenta cuando se realiza la operación en una paciente anciana (16). De acuerdo a esas informaciones, los médicos concordaron que la cirugía no era necesaria, una vez que se trataba de una paciente anciana y sin complicaciones, a pesar de los episodios recurrentes de dolor abdominal durante años. La paciente fue orientada en cuanto a posibles complicaciones y riesgos de la cirugía en la extracción del feto calcificado y optó por la mantención del feto en su abdomen. Actualmente la paciente está en buen estado general y asintomática, y en seguimiento ambulatorio en el Programa de Salud de la Familia. 


\section{REFERENCIAS}

1. Lin EP, Bhatt S, Dogra VS. Diagnostic clues to ectopic pregnancy. Radiographics 2008;28:1661-71.

2. Irick MB, Kitsos CN, O'Leary JA. Therapeutic aspects in the management of a lithopedion. Am Surg 1970;36:232-4.

3. Costa SD, Presley J, Bastert G. Advanced abdominal pregnancy. Obstet Gynecol Surv 1991;46:515-25.

4. Hincapie I, Navarro H, Mosquera J. Litopedion. Diagnóstico diferencial de tumor de ovário. Informe de um caso. Colombia Médica 1995;26:30-2.

5. Febronio EM, Rosas QG, Cardia PP, D'lppolito G. Gravidez ectópica: ensaio iconográfico com enfoque em achados de tomografia computadorizada e ressonância magnética. Radiol Bras 2012;45:279-82.

6. White RG. Advanced abdominal pregnancy - a review of 23 cases. Ir J Med Sci 1989;158:77-8.

7. Mbura JS, Mgaya HN. Advanced abdominal pregnancy in Muhimbili Medical Centre, Tanzania. Int J Gynecol Obstet1986;24:169-76.

8. Spiritos NM, Eisenkop SM, Mishell DR. Lithokelyphos: a case report and literature review. J Reprod Med 1987;32:43-6.
9. Rahman MS, Al Suleiman AS, Rahman J, Al Sibai $\mathrm{MH}$. Advanced abdominal pregnancy -observations in 10 cases. Obstet Gynecol 1982;59:366-72.

10. Rache JE, Cardozo MA, Sauer H, Weimann JO, Rache AS, Nardin MT. Abdome agudo obstrutivo causado por gestaçäo abdominal com litopédio "em formaçäo". Rev Bras Ginecol Obstet 1989;11(10):206-7.

11. Gonçalves C, Pimentel A, Leitão S, Santos RM, Costa JN. Caso Clínico: Litoquelifopédio. Acta Med Port 2011;24:621-4.

12. Pasini R, Knobel R, Parpinelli MA, Gonçalvez B, Amaral E, de Castro FG, de Araujo CR. Calcified abdominal pregnancy with eighteen years of evolution: case report. Sao Paulo Med J 2000;118:192-4.

13. Lachman N, Satyapal KS, Kalideen JM, et al. Lithopedion: A case report. Clin Anat 2001;14:52-4.

14. Zvandasara P. Advanced extrauterine pregnancy. Cent Afr J Med 1995;41:28-34.

15. Frayer CA, Hibbert ML. Abdominal pregnancy in a 67-year-old woman undetected for 37 years: a case report. J Reprod Med 1999;44:633-5.

16. Kim MS, Park S, Lee TS. Old abdominal pregnancy presenting as an ovarian neoplasm. J Korean Med Sci 2002;17: 274-5. 